\title{
Multi-variate Bateman method for two-body scattering without partial-wave decomposition
}

\author{
Zeki C. Kuruoğlu
}

Received: 13 September 2013 / Accepted: 28 March 2014 / Published online: 9 April 2014

(C) Springer International Publishing Switzerland 2014

\begin{abstract}
The use of Bateman method for solving the two-variable version of the twobody Lippmann-Schwinger equation without recourse to partial-wave decomposition is investigated. Bateman method is based on a special kind of interpolation of the momentum representation of the potential on a multi-variate grid. A suitable scheme for the generation of a multi-variate Cartesian grid is described. The method is tested on the Hartree potential for electron-hydrogen scattering in the static no-exchange approximation. Our results show that the Bateman method is capable of producing quite accurate solutions with relatively small number of grid points.
\end{abstract}

Keywords Quantum scattering theory · Lippmann-Schwinger equation · Few-body collisions - Multi-variate interpolation and approximation - Bateman interpolation . Degenerate-kernel methods for integral equations · Nystrom method ·

Faddeev equations

\section{Introduction}

The traditional approach to scattering problems has been through angular momentum decomposition. Recent years have seen a growing interest, especially in the context of few-body problems, for computational methods that avoid the decomposition of wave functions and scattering amplitudes into partial waves. Various direct multi-variable solution techniques for the two-body Lippmann-Schwinger (LS) equation have been investigated [1-10]. Most studies [1,3,5-9] employed the Nystrom method [11] which converts the multi-variable integral equation into a system of equations via a suitable multi-variate quadrature. The Nystrom method is capable of producing very accurate

Z. C. Kuruoğlu (ه)

Department of Chemistry, Bilkent University, 06800 Bilkent, Ankara, Turkey

e-mail: kuruoglu@bilkent.edu.tr 
results. However, the matrix dimensions in the multi-variable Nystrom approach can grow very fast to computationally prohibitive levels.

The off-shell two-body T-matrix is the essential input into the Faddeev equations $[12,13]$ for three-particle scattering. In the Faddeev approach to the three-atom problem, as formulated in Refs. [14-16], T-matrix elements for the diatomic potentials [17] have to be generated at a large number of energies and off-shell momenta. There is a growing awareness that, to solve Faddeev equations in momentum space, direct multi-variable approaches might be more appropriate than the traditional procedure of eliminating angular variables via partial-wave expansions $[1,18]$. In fact, in the context of nuclear three-body problems, considerable progress has been made towards the solution of Faddeev equations without invoking the angular-momentum decomposition [18-20]. Therefore, there is considerable interest in multi-variable methods that could produce the three-dimensional off-shell T-matrix in more economical manner than the Nystrom method. Galerkin [2,4], collocation [10], and Schwinger variational methods [10] have been investigated with various choices of multi-variable bases. These methods can effectively be viewed as contractions of the linear system of equations that the Nystrom approach gives rise to. In other words, the (large) equation system of the Nystrom method is replaced by a smaller set of approximate equations, by demanding that a residual vanishes on a chosen test space [10].

In this article we consider the Bateman method for solving the multi-variable integral equations of the two-body scattering problem. This method was originally proposed by Bateman [21] for the solution of single-variable integral equations. The bi-variate kernel of the integral equation is approximated by a separable expansion obtained from an interpolation of the bi-variate kernel on a grid. Its convergence and error bound has been studied in Refs. [22] and [23]. The best mean approximation for certain bi-variate kernels studied in Refs. [24,25] is in fact equivalent to the Bateman method. Bateman method involves two multi-variate grids: a primary grid for interpolation and a quadrature grid for evaluating the multi-dimensional integrals. It is interesting to note that a Nystrom calculation (with a given quadrature grid) can also be viewed as a Bateman calculation in which the same quadrature grid serves also as the interpolation grid.

Early discussions of the Bateman method in the context of few-body scattering calculations can be found in Refs. [26-30], where applications have been restricted to partial-wave (single-variable) LS equations. One notable exception is the work of Gianini and Lim [30] who applied the Bateman method to solve the two-variable LS equation for a Yukawa potential. Their application, however, suffered from limiting the interpolation grid to on-shell momenta and this prevented a true assessment of the full potential of the method.

The present paper aims to explore the potential utility of the Bateman method to solve the reduced (two-variable) Lippmann-Schwinger equation for two-body Tmatrix. Of course, it could also be applied to the full three-dimensional LS equation, but matrix dimensions arising from three-dimensional grids grow very quickly to require special computational platforms. At the two-variable level, Bateman calculations may be staged on commonly available computers. A finite-element like partitioning of the two-dimensional computational domain provides a sufficiently balanced interpolation grid for Bateman method to show its real potential. Our expectations of the Bateman 
method as a simple and practical tool are in fact borne out by the results of our test calculations on the Hartree potential, for which accurate scattering amplitudes at several energies are available in Ref. [2] from a finite-element solution of the twovariable Schrodinger equation.

Plan of this article is as follows: Sect. 2 introduces the notation and the LippmannSchwinger equation, as well as giving a brief review of the reduction of the threedimensional Lippmann-Schwinger equation into a two-dimensional integral equation. Sect. 3 discusses the basic structure of the Bateman method for the two-variable LS equation. Computational implementation is discussed in Sect. 4. In particular, the treatment of the singular integrals and the selection of the interpolation and quadrature grids is described. In Sect. 5, the results of Bateman calculations for the Hartree model are presented and compared with results of Ref. [2], and the benchmark Nystrom results of Ref. [10]. In Sect. 6, we summarize our conclusions.

Atomic units are used throughout this article.

\section{Lippmann-Schwinger equation}

The Lippmann-Schwinger (LS) equation for two-body scattering in operator form reads

$$
T(z)=V+V G_{0}(z) T(z)
$$

where $T$ is the transition operator, $V$ the two-body potential, $G_{0}=\left(z-H_{0}\right)^{-1}$, with $H_{0}$ being the free hamiltonian and $z$ the (complex) energy of the two-body system. Working in the center-of-mass frame, the eigenstates of $H_{0}$ are the relative momentum states $\mid \mathbf{q}>$, viz., $H_{0}\left|\mathbf{q}>=\left(q^{2} / 2 \mu\right)\right| \mathbf{q}>$. For on-shell scattering, $z=$ $E+i 0$, with $E=q_{0}^{2} / 2 \mu$, where $\mu$ is the reduced mass. The momentum-space matrix elements $T\left(\mathbf{q}, \mathbf{q}_{0} ; z\right)\left(\equiv<\mathbf{q}|T(z)| \mathbf{q}_{0}>\right.$ ) (referred to as the $T$-matrix) satisfy the three-dimensional integral equation

$$
T\left(\mathbf{q}, \mathbf{q}_{0} ; z\right)=V\left(\mathbf{q}, \mathbf{q}_{0}\right)+\int \mathrm{d} \mathbf{q}^{\prime} \frac{V\left(\mathbf{q}, \mathbf{q}^{\prime}\right) T\left(\mathbf{q}^{\prime}, \mathbf{q}_{0} ; z\right)}{z-q^{\prime 2} / 2 \mu}
$$

The $z$-dependence of the $T$-matrix elements $T\left(\mathbf{q}, \mathbf{q}_{0} ; z\right)$ will be suppressed, unless there is a need to explicitly show the energy dependence. The momentum-space representation $V\left(\mathbf{q}, \mathbf{q}^{\prime}\right)$ of the potential $V$ is given as

$$
V\left(\mathbf{q}, \mathbf{q}^{\prime}\right)=<\mathbf{q}|V| \mathbf{q}^{\prime}>=\int d \mathbf{r}<\mathbf{q}|\mathbf{r}>V(\mathbf{r})<\mathbf{r}| \mathbf{q}^{\prime}>
$$

with $<\mathbf{r} \mid \mathbf{q}>=e^{i \mathbf{r} \cdot \mathbf{q}} /(2 \pi)^{3 / 2}$. For central potentials, $V\left(\mathbf{q}, \mathbf{q}^{\prime}\right)$ and $T\left(\mathbf{q}, \mathbf{q}^{\prime}\right)$ depend only on $q, q^{\prime}$ and $x_{q q^{\prime}}$. Here, $x_{q q^{\prime}}$ denotes the cosine of the angle between vectors $\mathbf{q}$ and $\mathbf{q}^{\prime}$. We denote the polar and azimuthal angles of the momentum vectors $\mathbf{q}$ by $\theta$ and $\phi$, respectively. We then have $x_{q q^{\prime}}=\hat{\mathbf{q}} \cdot \hat{\mathbf{q}}^{\prime}=\cos \theta_{q q^{\prime}}=x x^{\prime}+s s^{\prime} \cos \left(\phi-\phi^{\prime}\right)$, where $x=\cos \theta$ and $s=\sqrt{1-x^{2}}$. To emphasize this functional dependence on $x_{q q^{\prime}}$, we will occasionally use the notation $T\left(q, q^{\prime}, x_{q q^{\prime}}\right)$ to stand for $T\left(\mathbf{q}, \mathbf{q}^{\prime}\right)$. 
For central potentials, the azimuthal-angle dependence in Eq. (2) can be integrated out to obtain a two-dimensional integral equation [1]. Towards this end, we introduce the averaged momentum states $\mid q x>$ via

$$
\left|q x>=(2 \pi)^{-1 / 2} \int_{0}^{2 \pi} d \phi\right| \mathbf{q}>=(2 \pi)^{-1 / 2} \int_{0}^{2 \pi} d \phi \mid q \theta \phi>.
$$

For a two-body operator $A$, we introduce reduced matrix elements by

$$
A\left(q, x ; q^{\prime}, x^{\prime}\right)=<q x|A| q^{\prime} x^{\prime}>=\int_{0}^{2 \pi} d \phi A\left(\mathbf{q}, \mathbf{q}^{\prime}\right)=\int_{0}^{2 \pi} d \phi A\left(q, q^{\prime}, x_{q q^{\prime}}\right) .
$$

For a rotationally invariant operator $A$, the above integral is independent of the variable $\phi^{\prime}$. Integrating Eq. (2) over $\phi$, we obtain the two-variable LS equation for the reduced $T$-matrix:

$$
\begin{aligned}
T\left(q, x ; q_{0}, x_{0}\right)= & V\left(q, x ; q_{0}, x_{0}\right) \\
& +2 \mu \int_{0}^{\infty} q^{\prime 2} d q^{\prime} \int_{-1}^{1} d x^{\prime} \frac{V\left(q, x ; q^{\prime}, x^{\prime}\right) T\left(q^{\prime}, x^{\prime} ; q_{0}, x_{0}\right)}{q_{0}^{2}-q^{\prime 2}+i 0} .
\end{aligned}
$$

If we take the initial momentum vector $\mathbf{q}_{0}$ along the $z$-axis, the half-off-shell $T$ matrix element for a general final momentum vector $\mathbf{q}_{f}$ is then given by

$$
<\mathbf{q}_{\mathbf{f}}|T| q_{0} \hat{\mathbf{z}}>=T\left(q_{f}, q_{0}, x_{f}\right)=(2 \pi)^{-1} T\left(q_{f}, x_{f} ; q_{0}, 1\right) .
$$

Direct numerical solution of this two-variable Lippmann-Schwinger (LS) equation without invoking the partial wave expansion can be performed nowadays in commonly available computational platforms. The approach used most frequently is the so-called Nystrom method [15] in which the integrals over $q^{\prime}$ and $x^{\prime}$ are approximated by a suitable two-variable quadrature and then $x$ and $q$ variables are collocated at the quadrature points, giving rise to a system of linear equations. Although the matrix dimension for the two-variable case is manageable and does not require special computing environment, going beyond two variables makes the matrix dimension quickly become computationally prohibitive. Therefore, in the contexts of three and four body problems, alternatives to Nystrom method would be welcome. Bateman method described in the next section is such an alternative.

\section{Bateman method}

Bateman method is based on a special kind of interpolation of $V\left(q, x ; q^{\prime}, x^{\prime}\right)$ on a finite set of grid points (nodes) in the $q-x$ computational domain $\left[0, q_{\max }\right] \times[-1,+1]$. Here, $q_{\max }$ is a momentum cutoff whose specification will be discussed in the next 
section. Bateman method involves an interpolation grid of $K$ distinct points in this computational domain. While there may be many different possibilities for choosing this bi-variate grid, we will use in this paper a cartesian grid.

Suppose two sets of nodes have been prescribed: $N$ distinct points $\left\{q_{1}, q_{2}, \ldots, q_{N}\right\}$ for $q$ in the interval $\left[0, q_{\max }\right]$ and $M$ distinct points $\left\{x_{1}, x_{2}, \ldots, x_{M}\right\}$ for $x$ in the interval $[-1,+1]$. The Cartesian product of these two sets generates a Cartesian grid of $K(=N M)$ points:

$$
\begin{aligned}
\mathcal{N} & =\left\{q_{1}, q_{2}, \ldots, q_{N}\right\} \times\left\{x_{1}, x_{2}, \ldots, x_{M}\right\} \\
& =\left\{\left(q_{n}, x_{m}\right): 1 \leq n \leq N, 1 \leq m \leq M\right\}
\end{aligned}
$$

The set $\mathcal{N}$ will be referred to as the interpolation grid. The Bateman interpolate $V^{B}\left(q, x ; q^{\prime}, x^{\prime}\right)$ of $V\left(q, x ; q^{\prime}, x^{\prime}\right)$ is defined as

$$
V^{B}\left(q, x ; q^{\prime}, x^{\prime}\right)=\sum_{n=1}^{N} \sum_{m=1}^{M} \sum_{n^{\prime}=1}^{N} \sum_{m^{\prime}=1}^{M} V\left(q, x ; q_{n}, x_{m}\right) \Lambda_{n m, n^{\prime} m^{\prime}} V\left(q_{n^{\prime}}, x_{m^{\prime}} ; q^{\prime}, x^{\prime}\right),
$$

where the matrix $\boldsymbol{\Lambda}$ is defined via

$$
\left(\boldsymbol{\Lambda}^{-1}\right)_{n m, n^{\prime} m^{\prime}}=V\left(q_{n}, x_{m} ; q_{n^{\prime}}, x_{m^{\prime}}\right)
$$

The (exact) transition operator $T^{B}$ for the separable potential $V^{B}$ is then given as

$$
T^{B}\left(q, x ; q^{\prime}, x^{\prime}\right)=\sum_{n=1}^{N} \sum_{m=1}^{M} \sum_{n^{\prime}=1}^{N} \sum_{m^{\prime}=1}^{M} V\left(q, x ; q_{n}, x_{m}\right) \mathbf{D}_{n m, n^{\prime} m^{\prime}} V\left(q_{n^{\prime}}, x_{m^{\prime}} ; q^{\prime}, x^{\prime}\right),
$$

where the matrix $\mathbf{D}$ is defined via

$$
\begin{aligned}
\left(\mathbf{D}^{-1}\right)_{n m, n^{\prime} m^{\prime}}= & <q_{n} x_{m}\left|V-V G_{0} V\right| q_{n^{\prime}} x_{m^{\prime}}>, \\
= & V\left(q_{n}, x_{m} ; q_{n^{\prime}} x_{m^{\prime}}\right) \\
& -2 \mu \int_{0}^{\infty} q^{\prime 2} d q^{\prime} \int_{-1}^{1} d x^{\prime} \frac{V\left(q_{n}, x_{m} ; q^{\prime}, x^{\prime}\right) V\left(q^{\prime}, x^{\prime} ; q_{n^{\prime}}, x_{m^{\prime}}\right)}{q_{0}^{2}-q^{\prime 2}+i 0} .
\end{aligned}
$$

This result corresponds to another instance of the weighted-residual approach [10] for the solution of the LS equation. It also follows from Schwinger variational method [31,32] if the wave function is expanded in the set of reduced momentum states $\left\{\mid q_{n} x_{m}>, n=1, \ldots, N, m=1, \ldots, M\right\}$. On the other hand, in the terminology of Ref. [33], $V^{B}$ is an inner-projection approximation of $V$.

We note in passing that the Bateman method could directly be used for the full threedimensional LS equation as well. In this case, we would choose $K$ distinct momentum vectors $\mathbf{q}_{k}$ in the computational domain $\left[0, q_{\max }\right] \times[-1,+1] \times[0,2 \pi]$ of variables $q, \cos \theta, \phi$. The Bateman approximation for the full T-matrix elements would then read 


$$
T^{B}\left(\mathbf{q}, \mathbf{q}^{\prime}\right)=\sum_{k=1}^{K} \sum_{k^{\prime}=1}^{K} V\left(\mathbf{q}, \mathbf{q}_{k}\right) \mathbf{D}_{k, k^{\prime}} V\left(\mathbf{q}_{\mathbf{k}^{\prime}}, \mathbf{q}^{\prime}\right),
$$

where

$$
\left(\mathbf{D}^{-1}\right)_{k, k^{\prime}}=<\mathbf{q}_{k}\left|V-V G_{0} V\right| \mathbf{q}_{k^{\prime}}>
$$

Here the inner-product is to be understood in its full three-dimensional sense.

\section{Computational implementation}

\subsection{Singular integrals}

To computationally implement the Bateman method, we need to introduce a quadrature rule on the $q-x$ computational domain for the evaluation of the matrix elements $<q_{n} x_{m}\left|V G_{0} V\right| q_{n^{\prime}} x_{m^{\prime}}>$. Again we opt for a direct-product quadrature scheme. Suppose $\left\{q_{\alpha}, \alpha=1, \ldots, N_{q}\right\}$ denote a suitable set of quadrature points for the $q$ variable, with corresponding weights $\left\{w_{\alpha}, \alpha=1, \ldots, N_{q}\right\}$. Similarly, let $\left\{x_{\beta}, \beta=\right.$ $\left.1, \ldots, N_{x}\right\}$ denote a set of quadrature points for the $x$-variable, with corresponding weights $\left\{\rho_{\beta}, \beta=1, \ldots, N_{x}\right\}$. A quadrature rule of order $N_{q} N_{x}$ is thus provided by the set $\left\{\left(q_{\alpha}, x_{\beta}\right)\right\}$ of quadrature points (referred to as the quadrature grid) and the set $\left\{\left(w_{\alpha}, \rho_{\beta}\right)\right\}$ of quadrature weights.

The evaluation of the matrix elements $<q_{n} x_{m}\left|V G_{0} V\right| q_{n^{\prime}} x_{m^{\prime}}>$ are carried out using essentially the same subtraction procedure described in detail in Ref. [10]. The singular integral is separated into its real and imaginary parts as

$$
<q_{n} x_{m}\left|V G_{0} V\right| q n^{\prime} x_{m^{\prime}}>=2 \mu A_{n m, n^{\prime} m^{\prime}}-i \pi \mu q_{0} B_{n m, n^{\prime} m^{\prime}}\left(q_{0}\right),
$$

where

$$
\begin{aligned}
A_{n m, n^{\prime} m^{\prime}} & =\mathcal{P} \int_{0}^{q_{\max }} d q \frac{q^{2} B_{n m, n^{\prime} m^{\prime}}(q)}{q_{0}^{2}-q^{2}} \\
B_{n m, n^{\prime} m^{\prime}}(q) & =\int_{-1}^{1} d x<q_{n} x_{m}|V| q x><q x|V| q_{n^{\prime}} x_{m^{\prime}}>,
\end{aligned}
$$

where $\mathcal{P}$ stands for principle-value integral. By adding and subtracting a singular integral that can be evaluated analytically, singular term $A_{n m, n^{\prime} m^{\prime}}$ is rearranged as a sum of non-singular and singular terms:

$$
A_{n m, n^{\prime} m^{\prime}}=A_{n m, n^{\prime} m^{\prime}}^{(n s)}+A_{n m, n^{\prime} m^{\prime}}^{(s)}
$$


where

$$
\begin{aligned}
& A_{n m, n^{\prime} m^{\prime}}^{(n s)}=\int_{0}^{q_{\max }} d q \frac{q^{2} B_{n m, n^{\prime} m^{\prime}}(q)-q_{0}^{2} B_{n m, n^{\prime} m^{\prime}}\left(q_{0}\right)}{q_{0}^{2}-q^{2}} \\
& A_{n m, n^{\prime} m^{\prime}}^{(s)}=B_{n m, n^{\prime} m^{\prime}}\left(q_{0}\right) \int_{0}^{q_{\max }} d q \frac{q_{0}^{2}}{q_{0}^{2}-q^{2}}=B_{n m, n^{\prime} m^{\prime}}\left(q_{0}\right) \frac{q_{0}}{2} \ln \frac{q_{\max }+q_{0}}{q_{\max }-q_{0}} .
\end{aligned}
$$

As the integrals involved in $B_{n m, n^{\prime} m^{\prime}}(q)$ and $A_{n m, n^{\prime} m^{\prime}}^{(n s)}$ are non-singular, they are amenable to approximation by quadrature with the result

$$
\begin{aligned}
B_{n m, n^{\prime} m^{\prime}}(q) & \approx \Sigma_{\beta=1}^{N_{x}} \rho_{\beta}<q_{n} x_{m}|V| q x_{\beta}><q x_{\beta}|V| q_{n^{\prime}} x_{m^{\prime}}> \\
A_{n m, n^{\prime} m^{\prime}} & \approx \Sigma_{\alpha=1}^{N_{q}} w_{\alpha} q_{\alpha}^{2} \frac{B_{n m, n^{\prime} m^{\prime}}\left(q_{\alpha}\right)}{q_{0}^{2}-q_{\alpha}^{2}}+C_{\text {sing }} q_{0}^{2} B_{n m, n^{\prime} m^{\prime}}\left(q_{0}\right)
\end{aligned}
$$

where

$$
C_{\text {sing }}=\frac{1}{2 q_{0}} \ln \frac{q_{\max }+q_{0}}{q_{\max }-q_{0}}-\sum_{\alpha=1}^{N_{q}} \frac{w_{\alpha}}{q_{0}^{2}-q_{\alpha}^{2}} .
$$

\subsection{Interpolation grid and quadrature}

To construct the Cartesian interpolation grid, we need to specify two uni-variate grids, one in $q$ and one in $x$. To define the $q$-grid $\left\{q_{n}\right\}$, the full $q$-domain is divided into two intervals: $\left[0,2 q_{0}\right]$, and $\left[2 q_{0}, \infty\right)$. This scheme is adopted to treat the singularity of the LS kernel at $q^{\prime}=q_{0}$ in Eqs. (2) or (6) as symmetrically as possible, and to have $q$-grid denser in the vicinity of $q_{0}$. To this end, the first interval $\left[0,2 q_{0}\right]$, is subdivided into $I_{1}$ (equal) subintervals (finite elements).

The second interval $\left[2 q_{0}, \infty\right)$, however, is first mapped to $[-1,+1]$ via the transformation

$$
u=\frac{q-2 q_{0}-f}{q-2 q_{0}+f}, \text { or } q=2 q_{0}+f \frac{1+u}{1-u},
$$

where $f$ is a scale factor. The $q$-variable is cut off at some large but finite value $q_{\max }$ by adopting an upper limit $u_{\max }(<1)$ to the variable $u$. In the calculations reported in this paper, we used $u_{\max }=0.99$, which corresponds to $q_{\max }$ values of several thousand in atomic units. This variable transformation is instrumental for a discretization of the semi-infinite interval $[0, \infty)$ with relatively few finite elements. The interval $\left[-1, u_{\max }\right]$ is divided into $I_{2}$ equal finite elements. Note that this gives rise to a non-uniform partitioning for the $q$-variable in the interval $\left[2 q_{0}, q_{\max }\right]$. The total number of finite elements covering the computational interval $\left[0, q_{\max }\right]$ is $I\left(\equiv I_{1}+I_{2}\right)$. The choice $I_{2}=3 I_{1}$ (hence $I=4 I_{1}$ ) was found adequate after some experimentation. The $q$-grid $\left\{q_{1}, q_{2}, \ldots, q_{N}\right\}$ consists of the end points and mid-points of this finiteelement partitioning. Note that $N=2 I+1, q_{1}=0$, and $q_{N}=q_{\max }$. 
The specification of the $x$-grid proceeds similarly to that of the $q$-grid. The interval $[-1,1]$ is partitioned into $J$ equal subintervals (finite-elements). Collecting and ordering the endpoints and midpoints of the finite elements together, we define the set of grid points $\left\{x_{1}, x_{2}, \ldots, x_{M}\right\}$, where $M=2 J+1, x_{0}=-1$ and $x_{M}=+1$.

To construct a composite Gauss-Legendre quadrature rule for $q$, each finite-element $\left[q_{2 i-1}, q_{2 i+1}\right], i=1,2, \ldots, I_{1}$ in the interval $\left[0,2 q_{0}\right]$, is mapped to $[-1,1]$ via $s=$ $\left(2 q-q_{2 i-1}-q_{2 i+1}\right) /\left(q_{2 i+1}-q_{2 i-1}\right)$. For finite elements in $\left[2 q_{0}, q_{\max }\right]$, we map the finite elements $\left[u_{2 i-1}, u_{2 i+1}\right]$, for $i=1, \ldots, I_{2}$, into $[-1,+1]$ via the map $s=(2 u-$ $\left.u_{2 i-1}-u_{2 i+1}\right) /\left(u_{2 i+1}-u_{2 i-1}\right)$. We choose a set of $n_{q}$ Gauss-Legendre quadrature points in the local variable $s$, and then transform them back to $q$-variable. The GaussLegendre quadrature points for all elements are then combined and ordered to form a composite quadrature rule with the set of quadrature points $\left\{q_{\alpha}, \alpha=1,2, \ldots, N_{q}\right\}$, where $N_{q}=I n_{q}$. The quadrature weights are similarly collected in the set $\left\{w_{\alpha}, \alpha=\right.$ $\left.1,2, \ldots, N_{q}\right\}$. In the calculations reported in the next section, values of $n_{q}$ ranged from 4 to 32 , depending on the fineness of the finite-element partitioning. To ensure results stable within 6-7 digits after the decimal point, the total number $N_{q}$ of quadrature points were typically in the order of 160-200, although 3-4 digit accuracy could be achieved with, say, $N_{q}=64$.

For the $x$-variable, each finite element $\left[x_{2 j-1}, x_{2 j+1}\right]$, is mapped to $[-1,+1]$ via the map $s=\left(2 x-x_{2 i-1}-x_{2 i+1}\right) /\left(x_{2 i+1}-x_{2 i-1}\right)$. We choose $n_{x}$ Gauss-Legendre quadrature points in $s$ and transform back to the $x$-variable. The quadrature points and their weights over individual finite elements are collected in the sets $\left\{x_{\beta}, \beta=\right.$ $\left.1,2, \ldots, N_{x}\right\}$, and $\left\{\rho_{\beta}, \beta=1,2, \ldots, N_{x}\right\}$, where $N_{x}=J n_{x}$. In our calculations, typically $N_{x}=80$ was sufficient to obtain results stable within 6 digits.

\section{Results}

To test the multi-variate Bateman method we used the Hartree potential

$$
V(r)=V_{0} e^{-\lambda r}\left(1+\frac{1}{r}\right) \text {. }
$$

The values used for the potential parameters are $V_{0}=-2.0$ and $\lambda=-2.0$, and the reduced mass is $\mu=0.5$. This potential corresponds to static no-exchange treatment of the electron-hydrogen scattering [2].

The momentum-space representation of the Hartree potential is given as

$$
V\left(\mathbf{q}, \mathbf{q}^{\prime}\right)=\frac{\lambda V_{0}}{\pi^{2}} \frac{1}{\left[\left(\mathbf{q}-\mathbf{q}^{\prime}\right)^{2}+\lambda^{2}\right]^{2}}-\frac{V_{0}}{2 \pi^{2}} \frac{1}{\left(\mathbf{q}-\mathbf{q}^{\prime}\right)^{2}+\lambda^{2}}
$$

For this potential the azimuthal integration in Eq. (4) can be carried out analytically to give

$$
\begin{aligned}
V\left(q, x ; q^{\prime}, x^{\prime}\right)= & \frac{2 \lambda V_{0}}{\pi} \frac{\left(q^{2}+q^{\prime 2}-2 q q^{\prime} x x^{\prime}+\lambda^{2}\right)}{\left[\left(q^{2}+q^{\prime 2}-2 q q^{\prime} x x^{\prime}+\lambda^{2}\right)^{2}-4 q^{2} q^{\prime 2}\left(1-x^{2}\right)\left(1-x^{\prime 2}\right)\right]^{3 / 2}} \\
& -\frac{V_{0}}{\pi} \frac{1}{\left[\left(q^{2}+q^{\prime 2}-2 q q^{\prime} x x^{\prime}+\lambda^{2}\right)^{2}-4 q^{2} q^{\prime 2}\left(1-x^{2}\right)\left(1-x^{\prime 2}\right)\right]^{1 / 2}}
\end{aligned}
$$


In situations where $V\left(q, x ; q^{\prime}, x^{\prime}\right)$ is not available analytically, it can be generated numerically by applying a suitable quadrature to the integral over the azimuthal angle $\phi$. In fact, this aspect was tested on the present potential. A composite 64-point GaussLegendre rule for the $\phi$-integral produced results that are indistinguishable within 7-8 digits from those of the analytical reduced potential.

Tables 1, 2, 3 and 4 show the convergence pattern of the Bateman results as the number of grid points in $q$ and $x$ variables are increased. Two collision energies considered are $E=0.25$ and $E=4.0$. Shown are the real and imaginary parts of the scattering amplitude

$$
A(x ; E) \equiv-4 \pi^{2} \mu T\left(q_{0}, x ; q_{0}, x_{0}=1.0 ; E\right)
$$

for three values of $x$. Also shown is the s-wave component of the scattering amplitude, obtained by numerically averaging $A(x, E)$ over $x$.

Reference values in these tables were obtained using $N_{q}=200$ and $N_{x}=80$ in the Nystrom method. These are stable to within at least the number of digits shown against further increases in the computational parameters like $N_{q}, N_{x}, q_{\max }$ and against the

Table 1 Convergence of the Bateman method with respect to the number of interpolation points in the $q$-grid

Shown are the scattering amplitudes $A(x ; E)$ at $E=0.25$. Parameters $N$ and $M$ denote the number of points in the $q$ - and $x$-grids, respectively

\begin{tabular}{|c|c|c|c|c|c|}
\hline$N$ & $M$ & s-wave & $\mathrm{x}=1.0$ & $\mathrm{x}=0.0$ & $\mathrm{x}=-1.0$ \\
\hline & & \multicolumn{4}{|c|}{ Real part of scattering amplitude } \\
\hline 9 & 41 & 0.869160 & 1.040426 & 0.862265 & 0.725922 \\
\hline 17 & 41 & 0.868613 & 1.039856 & 0.861694 & 0.725350 \\
\hline 25 & 41 & 0.868570 & 1.039813 & 0.861651 & 0.725307 \\
\hline 33 & 41 & 0.868561 & 1.039804 & 0.861641 & 0.725297 \\
\hline 41 & 41 & 0.868557 & 1.039800 & 0.861638 & 0.725294 \\
\hline 49 & 41 & 0.868556 & 1.039799 & 0.861637 & 0.725292 \\
\hline 65 & 41 & 0.868555 & 1.039798 & 0.861635 & 0.725291 \\
\hline 81 & 41 & 0.868554 & 1.039797 & 0.861635 & 0.725291 \\
\hline 81 & 51 & 0.868553 & 1.039796 & 0.861634 & 0.725290 \\
\hline \multicolumn{2}{|c|}{ Nystrom } & 0.868552 & 1.039795 & 0.861633 & 0.725289 \\
\hline \multirow{2}{*}{\multicolumn{2}{|c|}{ Ref. [2] }} & 0.869 & 1.040 & 0.862 & 0.725 \\
\hline & & \multicolumn{4}{|c|}{ Imaginary part of scattering amplitude } \\
\hline 9 & 41 & 1.494530 & 1.498614 & 1.494521 & 1.490485 \\
\hline 17 & 41 & 1.495490 & 1.499574 & 1.495481 & 1.491445 \\
\hline 25 & 41 & 1.495566 & 1.499650 & 1.495557 & 1.491521 \\
\hline 33 & 41 & 1.495583 & 1.499667 & 1.495573 & 1.491538 \\
\hline 41 & 41 & 1.495589 & 1.499673 & 1.495579 & 1.491544 \\
\hline 49 & 41 & 1.495589 & 1.499675 & 1.495582 & 1.491546 \\
\hline 65 & 41 & 1.495593 & 1.499677 & 1.495584 & 1.491548 \\
\hline 81 & 41 & 1.495594 & 1.499678 & 1.495585 & 1.491549 \\
\hline 81 & 51 & 1.495596 & 1.499680 & 1.495586 & 1.491550 \\
\hline \multicolumn{2}{|c|}{ Nystrom } & 1.495598 & 1.499682 & 1.495589 & 1.491553 \\
\hline \multicolumn{2}{|c|}{ Ref. [2] } & 1.495 & 1.500 & 1.496 & 1.491 \\
\hline
\end{tabular}


Table 2 Convergence of the Bateman method with respect to the number of interpolation points in the $x$-grid

Shown are the scattering amplitudes $A(x ; E)$ at $E=0.25$. Parameters $N$ and $M$ denote the number of points in the $q$ - and $x$-grids, respectively

\begin{tabular}{lllll}
\hline$N \quad M \quad$ s-wave & $\mathrm{x}=1.0$ & $\mathrm{x}=0.0$ & $\mathrm{x}=-1.0$ \\
\hline
\end{tabular}

Real part of scattering amplitude

$\begin{array}{ll}41 & 11 \\ 41 & 15 \\ 41 & 21 \\ 41 & 25 \\ 41 & 31 \\ 41 & 41 \\ 41 & 51\end{array}$

$0.868724 \quad 1.039964$

0.861805

0.725461

$0.868611 \quad 1.039854$

0.861692

0.725348

$0.868572 \quad 1.039816$

0.861653

0.725309

$\begin{array}{lll}0.868564 & 1.039808 & 0.861646\end{array}$

0.725302

$\begin{array}{lll}0.868560 & 1.039803 & 0.861641\end{array}$

0.725297

$0.868557 \quad 1.039800$

0.861638

0.725294

Nystrom

$0.868556 \quad 1.039799$

0.861637

0.725293

$0.868552 \quad 1.039795$

0.861633

0.725289

Imaginary part of scattering amplitude

\begin{tabular}{llllll}
41 & 11 & 1.495293 & 1.499375 & 1.495284 & 1.491246 \\
41 & 15 & 1.495493 & 1.499576 & 1.495484 & 1.491447 \\
41 & 21 & 1.495562 & 1.499645 & 1.495552 & 1.491516 \\
41 & 25 & 1.495575 & 1.499659 & 1.495566 & 1.491530 \\
41 & 31 & 1.495584 & 1.499668 & 1.495574 & 1.491539 \\
41 & 41 & 1.495589 & 1.499673 & 1.495579 & 1.491544 \\
41 & 51 & 1.495591 & 1.499674 & 1.495581 & 1.491545 \\
Nystrom & 1.495598 & 1.499682 & 1.495589 & 1.491553 \\
\hline
\end{tabular}

Table 3 Convergence of the Bateman method with respect to the number of interpolation points in the $q$-grid

Shown are the scattering amplitudes $A(x ; E)$ at $E=4.0$. Parameters $N$ and $M$ denote the number of points in the $q$ - and $x$-grids, respectively

\begin{tabular}{|c|c|c|c|c|c|}
\hline$N$ & $M$ & s-wave & $\mathrm{x}=1.0$ & $\mathrm{x}=0.0$ & $\mathrm{x}=-1.0$ \\
\hline & & \multicolumn{4}{|c|}{ Real part of scattering amplitude } \\
\hline 9 & 41 & 0.244835 & 0.976089 & 0.161554 & 0.0507304 \\
\hline 17 & 41 & 0.245864 & 0.978461 & 0.162482 & 0.0508273 \\
\hline 25 & 41 & 0.245917 & 0.978598 & 0.162528 & 0.0508275 \\
\hline 33 & 41 & 0.245919 & 0.978603 & 0.162530 & 0.0508274 \\
\hline 41 & 41 & 0.245919 & 0.978604 & 0.162530 & 0.0508274 \\
\hline 41 & 51 & 0.245919 & 0.978604 & 0.162530 & 0.0508274 \\
\hline \multicolumn{2}{|c|}{ Nystrom } & 0.245919 & 0.978604 & 0.162530 & 0.0508275 \\
\hline \multirow{2}{*}{\multicolumn{2}{|c|}{ Ref. [2] }} & 0.246 & 0.979 & 0.164 & 0.051 \\
\hline & & \multicolumn{4}{|c|}{ Imaginary part of scattering amplitude } \\
\hline 9 & 41 & 0.199444 & 0.292994 & 0.191600 & 0.139506 \\
\hline 17 & 41 & 0.204711 & 0.298829 & 0.196851 & 0.144271 \\
\hline 25 & 41 & 0.205001 & 0.299153 & 0.197139 & 0.144531 \\
\hline 33 & 41 & 0.205013 & 0.299167 & 0.197151 & 0.144541 \\
\hline 41 & 41 & 0.205013 & 0.299167 & 0.197151 & 0.144542 \\
\hline 41 & 51 & 0.205014 & 0.299168 & 0.197152 & 0.144542 \\
\hline \multicolumn{2}{|c|}{ Nystrom } & 0.205014 & 0.299168 & 0.197152 & 0.144543 \\
\hline \multicolumn{2}{|c|}{ Ref. [2] } & 0.205 & 0.300 & 0.197 & 0.145 \\
\hline
\end{tabular}


Table 4 Convergence of the Bateman method with respect to the number points in the $x$-grid

Shown are the scattering amplitudes $A(x ; E)$ at $E=4.0$. Parameters $N$ and $M$ denote the number of points in the $q$ - and $x$-grids, respectively

\begin{tabular}{lrllll}
\hline$N$ & $M$ & s-wave & $\mathrm{x}=1.0$ & $\mathrm{x}=0.0$ & $\mathrm{x}=-1.0$ \\
\hline \multicolumn{5}{c}{ Real part of scattering amplitude } \\
41 & 11 & 0.245890 & 0.978503 & 0.162522 & 0.0508334 \\
41 & 15 & 0.245910 & 0.978572 & 0.162525 & 0.0508253 \\
41 & 21 & 0.245917 & 0.978597 & 0.162528 & 0.0508264 \\
41 & 25 & 0.245918 & 0.978601 & 0.162529 & 0.0508269 \\
41 & 31 & 0.245919 & 0.978603 & 0.162530 & 0.0508272 \\
41 & 41 & 0.245919 & 0.978604 & 0.162530 & 0.0508274 \\
41 & 51 & 0.245919 & 0.978604 & 0.162530 & 0.0508274 \\
Nystrom & 0.245919 & 0.978604 & 0.162530 & 0.0508275 \\
& & Imaginary & part of scattering amplitude \\
41 & 11 & 0.204881 & 0.298973 & 0.197048 & 0.144436 \\
41 & 15 & 0.204980 & 0.299120 & 0.197123 & 0.144511 \\
41 & 21 & 0.205006 & 0.299157 & 0.197144 & 0.144534 \\
41 & 25 & 0.205010 & 0.299163 & 0.197148 & 0.144539 \\
41 & 31 & 0.205012 & 0.299166 & 0.197151 & 0.144541 \\
41 & 41 & 0.205013 & 0.299167 & 0.197152 & 0.144542 \\
41 & 51 & 0.205014 & 0.299168 & 0.197152 & 0.144542 \\
Nystrom & 0.205014 & 0.299168 & 0.197152 & 0.144543 \\
\hline \multicolumn{7}{c}{} & & & &
\end{tabular}

variations in the the distribution pattern of quadrature points in the $q-x$ plane. Also shown in Tables 1 and 3 are the results of Ref. [2], obtained via a finite element solution of two-variable Schrodinger equation for the same model.

Matrix dimensions (or number of grid points) for Bateman calculations reported in Table 1 range from 369 to 4,131, while the reference Nystrom calculation involved a kernel matrix dimension of 16,000 . An examination of these tables show that results accurate to 3-4 digits can be obtained with relatively few grid points. However, going beyond this level of accuracy may require much finer interpolation grids. Nevertheless, even with fairly dense interpolation grids, there is considerable reduction in the matrix size.

One can argue that whenever the number of interpolation points are comparable to the number of quadrature points, the Bateman and Nystrom methods will yield similar levels of accuracy. In fact, as mentioned earlier, when a quadrature grid is also used as interpolation grid, Bateman method degenerates into the Nystrom method. Conversely, a Nystrom calculation is at the same time a Bateman calculation. In a sense, Nystrom is a restricted type of Bateman method in which interpolation and quadrature grids are the same. That Bateman approach distinguishes between interpolation and quadrature grids is a strength of the Bateman approach over the Nystrom approach. For a given (crude) interpolation grid, the integral involved in the matrix element $<q_{n} x_{m}\left|V G_{0} V\right| q_{n^{\prime}} x_{m^{\prime}}>$ can be calculated with a finer quadrature grid, without affecting the the order of the matrix $\mathbf{D}$. In contrast, in Nystrom method, the quadrature grid used to discretize the integral will have to be used also as collocation points in order to obtain a consistent set of equations. 


\section{Discussion and conclusions}

We have shown that the multi-variate Bateman interpolation of $V\left(q, x ; q^{\prime}, x^{\prime}\right)$ on a grid provide a simple and viable computational scheme to solve the LS equation without invoking angular momentum decomposition. In terms of computational complexity, Bateman approach is quite comparable to the Nystrom method. Although more computing time is needed to form the $\mathbf{D}^{-1}$-matrix than to assemble the kernel matrix of the Nystrom method, Bateman method (and other weighted residual methods) represent a contraction of the system of equations of the Nystrom method. In fact, when interpolation and quadrature grids are taken to coincide, Bateman and Nystrom methods become equivalent.

The use of two grids in the Bateman approach, one for interpolation and one for quadrature evaluation of matrix elements, gives it an additional flexibility. The matrix dimension is determined by the interpolation grid. However, calculation of the integral in Eq. (12) for the construction of the matrix $\mathbf{D}^{-1}$ can be carried out with a higher-order quadrature rule without affecting the matrix size. From this point of view, Nystrom approach is a restricted type of Bateman method with the same grid used for the purposes of interpolation and quadrature both.

We find that Bateman method can yield 3-4 digit accuracy with relatively small numbers of interpolation points. However, as the size of the interpolation grid is increased to achieve higher level of accuracy, the advantage associated with the reduction in matrix dimension may disappear to some extent. With large sets of interpolation and quadrature points, both Nystrom and Bateman methods are expected to perform satisfactorily.

In three- and four-particle contexts, the two-particle $T$-matrix $<\mathbf{q}|T(E)| \mathbf{q}^{\prime}>$ is needed at very many different two-particle energies $E$ and for many different off-shell momenta $\mathbf{q}$ and $\mathbf{q}^{\prime}$. Bateman method could be an effective way of generating arbitrary off-shell $T$-matrix elements needed in direct momentum-vector approaches to solve three-particle Faddeev equations without employing partial-wave decomposition as in Refs.[19,20].

Acknowledgments The author gratefully acknowledges the computation time provided by Prof. B. Tanatar on the computing facility of his research group in the Department of Physics at Bilkent University.

\section{References}

1. Ch. Elster, J.H. Thomas, W. Glöckle, Two-body T-matrices without angular-momentum decomposition: energy and momentum dependences. Few-Body Syst. 24, 55 (1998)

2. J. Shertzer, A. Temkin, Direct calculation of the scattering amplitude without-partial wave analysis. Phys. Rev. A 63, 062714 (2001)

3. G.L. Caia, V. Pascalutsa, L.E. Wright, Solving potential scattering equations without partial wave decomposition. Phys. Rev. C 69, 034003 (2004)

4. B.M. Kessler, G.L. Payne, W.N. Polyzou, Application of wavelets to singular integral scattering equations. Phys. Rev. C 70, 034003 (2004)

5. A.S. Kadyrov, I. Bray, A.T. Stelbovics, B. Saha, Direct solution of the three-dimensional LippmannSchwinger equation. J. Phys. B 38, 509 (2005)

6. G. Ramalho, A. Arriaga, M.T. Peña, Solution of the spectator equation for relativistic NN scattering without partial wave expansion. Few-Body Syst. 39, 123 (2006) 
7. M. Rodríguez-Gallardo, A. Deltuva, E. Cravo, R. Crespo, A.C. Fonseca, Two-body scattering without angular-momentum decomposition. Phys. Rev. C 78, 034602 (2008)

8. M. Rodríguez-Gallardo, A. Deltuva, E. Cravo, R. Crespo, A.C. Fonseca, Two-body scattering without angular-momentum decomposition: fully off-shell T-matrices. Eur. Phys. J. A42, 601 (2009)

9. A.S. Kadyrov, I.B. Abdurakhmanov, I. Bray, A.T. Stelbovics, Three-dimensional integral-equation approach to proton- and antiproton-hydrogen collisions. Phys. Rev. A 80, 022704 (2009)

10. Z.C. Kuruoğlu, Weighted-residual methods for the solution of two-particle Lippmann-Schwinger equation without partial-wave decomposition. Few-Body Syst. 55, 69 (2014)

11. K.E. Atkinson, A Survey of Numerical Methods for the Solution of Fredholm Integral Equations of the Second Kind (SIAM, Philadelphia, 1976)

12. L.D. Faddeev, S.P. Merkuriev, Quantum Scattering Theory for Several Particle Systems (Kluwer, Dordrecht, 1993)

13. W. Glöckle, The Quantum Mechanical Few-Body Problem (Springer, Berlin, 1983)

14. Z.C. Kuruoğlu, D.A. Micha, Collision dynamics of three interacting atoms: the Faddeev equations in a diabatic electronic basis. J. Chem. Phys. 79, 6115 (1983)

15. Z.C. Kuruoğlu, D.A. Micha, Collision dynamics of three interacting atoms: model calculations of $\mathrm{H}+$ $\mathrm{H}_{2}$ resonances. J. Chem. Phys. 80, 4262 (1984)

16. Z.C. Kuruoğlu, D.A. Micha, Calculation of resonances in the $\mathrm{H}+\mathrm{H}_{2}$ reaction using the Faddeev-AGS method. Int. J. Quantum Chem. S23, 105 (1989)

17. Z.C. Kuruoğlu, D.A. Micha, Diatomic transition operators: results of L2-basis expansions. J. Chem. Phys. 72, 3328 (1980)

18. W. Schadow, Ch. Elster, W. Glöckle, Three-body scattering below breakup threshold: an approach without using partial waves. Few-Body Syst. 28, 15 (2000)

19. H. Liu, Ch. Elster, W. Glöckle, Three-body scattering at intermediate energies. Phys. Rev. C 72, 054003 (2005)

20. Ch. Elster, W. Glöckle, H. Witała, A new approach to the 3D Faddeev equation for three-body scattering. Few-Body Syst. 45, 1 (2009)

21. H. Bateman, On the numerical solution of linear integral equations. Proc. R. Soc. Lond. A 100, 441 (1922)

22. G.T. Thompson, On Bateman's method for solving linear integral equations. J. Assoc. Comput. Mach. 4, 314 (1957)

23. S. Joe, I.H. Sloan, On Bateman's method for second kind integral equations. Numer. Math. 49, 499 (1986)

24. C.A. Micchelli, A. Pinkus, Best mean approximation to a 2-dimensional kernel by tensor products. Bull. Am. Math. Soc. 83, 400 (1977)

25. E.W. Cheney, Multivariate Aprroximation Theory: Selected Topics (SIAM, Philadelphia, 1986)

26. V.B. Belyaev, A.L. Zubarev, Some possibilities of solving the Faddeev equations with potentials of arbitrary form. Sov. J. Nucl. Phys. 14, 305 (1972)

27. V.I. Kukulin, Algebraic reduction of the Faddeev equations. Sov. J. Nucl. Phys. 14, 481 (1972)

28. S. Oryu, Generalized separable potential theory and Bateman's method on the scattering problem. Prog. Theor. Phys. 52, 550 (1974)

29. V.B. Belyaev, E. Wrzecionko, B.F. Irgaziev, Solution of three-dimensional Lippmann-Schwinger equation without partial-wave expansion. Sov. J. Nucl. Phys. 20, 664 (1975)

30. T.K. Lim, J. Giannini, Separable-expansion method for potential scattering and the off-shell T-matrix. Phys. Rev. A 18, 517 (1978)

31. A.L. Zubarev, Schwinger variational principle. Sov. J. Part. Nucl. 9, 188 (1978)

32. S.K. Adhikari, Variational Principles and the Numerical Solution of Scattering Problems (Wiley, New York, 1998)

33. P.O. Löwdin, Linear Algebra for Quantum Theory (Wiley, New York, 1998) 